\title{
Accretion Disks around Black Holes with Advection and Optical Depth Transition
}

\author{
Yulia V. Artemova ${ }^{1}$, Gennadi S. Bisnovatyi-Kogan ${ }^{1,2}$ \\ ${ }^{1}$ Space Research Institute, Profsoyuznaya 84/32, Moscow 117997, Russia; \\ julia@iki.rssi.ru, gkogan@iki.rssi.ru \\ ${ }^{2}$ Joint Institute Nuclear Research, Dubna, Russia \\ Igor V. Igumenshchev ${ }^{3}$ \\ ${ }^{3}$ Laboratory for Laser Energetics, University of Rochester, \\ 250 East River Road, Rochester, NY 14623, USA ; iigu@lle.rochester.edu \\ Igor D. Novikov ${ }^{4,5}$ \\ ${ }^{4}$ Niels Bohr Institute, Blegdamsvej 17, DK-2100, Copenhagen, Denmark; novikov@nbi.dk \\ ${ }^{5}$ Astro-Space Center of P.N. Lebedev Physical Institute, Profsouznaya 84/32, Moscow 117997, Russia
}

\begin{abstract}
We consider the effects of advection and radial gradients of pressure and radial drift velocity on the structure of accretion disks around black holes with proper description of optically thick/thin transitions. We concentrated our efforts on the models with large accretion rate. Contrary to disk models neglecting advection, we find that continuous solutions extending from the outer disk regions to the inner edge exist for all accretion rates we have considered. We show that the sonic point moves outward with increasing accretion rate, and that in the innermost disk region advection acts as a heating process that may even dominate over dissipative heating. Despite the importance of advection on it's structure, the disk remains geometrically thin. Global solutions of advective accretion disks, which describe continuously the transition between optically thick outer region and optically thin inner region are constructed and analyzed.
\end{abstract}

Subject headings: Accretion, accretion disks — black hole physics — hydrodynamics

\section{Introduction}

The "standard accretion disk model" of Shakura (1972) and Shakura \& Sunyaev (1973), that has been widely used to model accretion flows around black holes, is based on a number of simplifying assumptions. In particular, the flow is assumed to be geometrically thin and with a Keplerian angular velocity distribution. This assumption allows gradient terms in the differential equations describing the flow to be neglected, reducing them to a set of algebraic equations, and thereby fixes the angular momentum distribution of the flow. For low accretion rates, $\dot{M}$, this assumption is generally considered to be reasonable. Since the end of the seventies, however, it has been realized that for high accretion rates, advection of energy with the flow can crucially modify the properties of the innermost parts of accretion disks around black holes. A deviation from a Keplerian rotation may result.

Initial attempts to solve the more general disk problem only included advection of energy and the radial gradient of pressure in models with small values of the viscosity parameter, $\alpha=10^{-3}$ (Paczyński \& Bisnovatyi-Kogan 1981), and it was shown that including radial velocity in the radial momentum equation would not change principally the results for such a small $\alpha$ (Muchotrzeb \& Paczyński 1982). 
Liang \& Thomson (1980) emphasised the importance of the transonic nature of the radial drift velocity, and the influence of viscosity on the transonic accretion disk solutions was noted by Muchotrzeb (1983), who claimed that such solutions only existed for viscosity parameters smaller than $\alpha_{*} \simeq 0.02-0.05$. Matsumoto et al. (1984), then showed that solutions with $\alpha>\alpha_{*}$ do in fact exist, but the nature of the singular point, where the radial velocity equals the sound velocity, is changing from a saddle to a nodal type and the position of this point is shifted substantially outwards in the disk. Matsumoto et al. (1984) also demonstrated the non-uniqueness of the solutions with a nodal type critical point for given Keplerian boundary conditions at the outer boundary of the disk (see also Muchotrzeb-Czerny 1986). Extensive investigation of accretion disk models with advection for a wide range of the disk parameters, $\dot{M}$ and $\alpha$, was conducted by Abramowicz et al. (1988), with special emphasis on low $\alpha$. Misra \& Melia (1996) considered optically thin two-temperature disk models and treated advection in the framework of the Keplerian disk model, but fixed the proton temperature somewhat arbitrarily at the outer boundary. Chakrabarti (1996) solved the advection problem containing shock waves near the innermost disk region, considering accretion through saddle points. Numerical solutions of accretion disks with advection have been obtained by Chen and Taam (1993) for the optically thick case with $\alpha=0.1$, and for the optically thin case (Narayan 1996). A simplified account of advection has recently been attempted, either treating it like an additional algebraic term assuming a constant radial gradient of entropy (Abramowicz et al. 1995; Chen et al. 1995; Chen 1995), or using the condition of self-similarity (Narayan \& Yi 1994).

Over the last few years it has become clear, that neglecting the advective heat transport at high $\dot{M}$ leads to qualitatively wrong conclusions about the topology of the family of solutions of the disk structure equations (see for example Abramowicz et al. 1995; Chen et al. 1995; Artemova et al. 1996). The disk structure equations without advection give rise two branches of solutions: optically thick and optically thin, which do not intersect if $\dot{M}<\dot{M}_{c r} \approx(0.6-0.9) \dot{M}_{\text {Edd }}$ for $\alpha=1$ and $M_{B H}=10^{8} M_{\odot}$, where $\dot{M}_{\text {Edd }}$ is the Eddington accretion rate (Artemova et al. 1996). For larger accretion rates there are no solutions of these equations extending continuously from large to small radii, and with Keplerian boundary conditions at the outer boundary of the disk (see also Liang \& Wandel 1991; Wandel \& Liang 1991; Luo \& Liang 1994). It was argued by Artemova et al. (1996), that for accretion rates larger than $\dot{M}_{c r}$ advection becomes critically important and would allow solutions extending all the way to the inner disk edge also to exist for $\dot{M}>\dot{M}_{c r}$.

The consistent transonic solutions for optically thick advective accretion disks were constructed in a wide range of values of the viscosity parameter $\alpha$ and mass accretion rate (see Artemova et al. (2001)) and character of singular points of obtained solutions was investigated. However for some values of mass accretion rate and viscosity parameter the corresponding value of optical depth in the inner region of accretion disk becomes less than unity and the used assumptions for optically thick disk approximation are violated. It requires to include into system of equations the transition formulae for radiative pressure and radiative flux (Artemova et al., 1996) to describe correctly the intermediate region between optically thick and optically thin zone.

The goal of the present paper is to construct explicitly accretion disk models with optical depth transition for high $\dot{M}$ taking advective heat transport self-consistently into account. We also include radial gradients of pressure and radial drift velocity and we allow for the non-Keplerian character of the circular velocity. Furthermore, we use the geometrically thin disk approximation because, the relative thickness of the disk in the considering models is less than unity. We show that solutions extending from large radii to the inner edge of the disk can be constructed even for accretion rates considerably larger than $\dot{M}_{c r}$ and the smooth transition between optically thick and optically thin regions in accretion disk appeares when the value of mass accretion rate becomes close to the critical one for considering models. We find that 
advection is very important in the innermost disk region.

In $\S 2$ we introduce our model and solution methods, while in $\S 3$ we discuss our results.

\section{The Model and the Method of Solution}

In this paper we will consider the full set of solutions to the disk structure equations with advection including the optically thick, optically thin and the intermediate zones in accretion disks.

In our models we used two differents prescriptions for viscosity

$$
t_{r \phi}=-\alpha P
$$

suggested by Shakura (1972) and the angular velocity gradient-dependent viscous stress,

$$
t_{r \phi}=\rho \nu r \frac{d \Omega}{d r},
$$

where $\nu$ is the kinematic viscosity coefficient.

We use from now on geometric units with $G=1, c=1$, use $r$ as the radial coordinate scaled to $r_{g}=M$, and scale all velocities to $c$. We work with the pseudo-Newtonian potential proposed by Paczyński and Wiita (1980), $\Phi=-M /(r-2)$, that provides an accurate, yet simple approximation to the Schwarzschild geometry. We normalize the accretion rate as $\dot{m}=\dot{M} / \dot{M}_{\mathrm{Edd}}$, where $\dot{M}_{\mathrm{Edd}}=L_{\mathrm{Edd}}=4 \pi M m_{p} / \sigma_{T}$, in our units.

We use the same set of equations, ingredients and boundary conditions in our models as in the paper of Artemova et al. (2001)), except for changes required by the formulae for radiative flux and radiation pressure to describe correctly the intermediate zone in the accretion disk at high accretion rates. We used the same numerical method as Artemova et al. (2001) to solve this modified system of algebraic and differential equations.

The following equations are therefore modified:

The vertically averaged energy conservation equation:

$$
Q_{a d v}=Q^{+}-Q^{-}
$$

where

$$
\begin{gathered}
Q_{a d v}=-\frac{\dot{M}}{4 \pi r}\left[\frac{d E}{d r}+P \frac{d}{d r}\left(\frac{1}{\rho}\right)\right], \\
Q^{+}=-\frac{\dot{M}}{4 \pi} r \Omega \frac{d \Omega}{d r}\left(1-\frac{l_{\text {in }}}{l}\right), \\
Q^{-}=\frac{2 a T^{4} c}{3 \kappa \rho h}\left(1+\frac{4}{3 \tau_{0}}+\frac{2}{3 \tau_{*}^{2}}\right)^{-1},
\end{gathered}
$$

are the advective energy, the viscous dissipation rate and the cooling rate per unit surface, respectively, $T$ is the midplane temperature, $\kappa$ is the opacity, $a$ is the radiation constant and $\tau_{0}$ is the Thompson optical depth, $\tau_{0}=\kappa \rho h$. Here we have introduced the total optical depth to absorption, $\tau_{\alpha} \ll \tau_{0}$,

$$
\tau_{\alpha} \simeq 5.2 * 10^{21} \frac{\rho^{2} T^{1 / 2} h}{a c T^{4}}
$$


and the effective optical depth

$$
\tau_{*}=\left(\tau_{0} \tau_{\alpha}\right)^{1 / 2} .
$$

Where $\rho$ is the density and $h$ is the half-thickness of the disk.

The equation of state for the matter consisted of a gas-radiation mixture is

$$
P_{\text {tot }}=P_{\text {gas }}+P_{\text {rad }},
$$

where the gas pressure is given by

$$
P_{\text {gas }}=\rho \mathcal{R} T,
$$

where $\mathcal{R}$ is the gas constant.

The expression for the radiation pressure is

$$
P_{\text {rad }}=\frac{a T^{4}}{3}\left(1+\frac{4}{3 \tau_{0}}\right)\left(1+\frac{4}{3 \tau_{0}}+\frac{2}{3 \tau_{*}^{2}}\right)^{-1} .
$$

In our calculations we use the dimensionless parameter $\beta$ to describe the relation of gas pressure to the total pressure in accretion disk

$$
\beta=\frac{P_{\text {gas }}}{P_{\text {gas }}+P_{\text {rad }}}
$$

The specific energy of the mixture is

$$
\rho E=\frac{3}{2} P_{\text {gas }}+3 P_{\text {rad }} .
$$

Our method allows us to construct a self-consistent solution to the system of equations from very large radii, $r>>100$, and down to the innermost regions of the disk.

\section{Results and Discussion}

We will now compare the solutions with advection and without it. In the "standard model", for accretion rates $\dot{m}<\dot{m}_{c r}=36$, (for $\alpha=0.5$ and $M_{B H}=10 M_{\odot}$, where $\dot{m}_{c r}$ is the critical accretion rate) there always exist solutions that extend continuously from large to small radii. When $\dot{m}>\dot{m}_{c r}=36$ there are no solutions in a range of radii around $r \approx 13$, and therefore no continuous solutions extending from large radii to the innermost disk edge (see detailed discussion by Artemova et al. 1996, where however, the Newtonian potential was used, resulting in $\dot{m}_{c r}=9.4$ for $\alpha=1.0$ and $M_{B H}=10^{8} M_{\odot}$ ).

In Figure 1 and Figure 2 we plot the Thomson scattering depth $\tau_{0}$ and the effective optical depth $\tau_{*}$ as a function of radius, $r$, for different $\dot{m}$ (for $\alpha=0.5$ and $M_{B H}=10 M_{\odot}$ ), clearly demonstrating that the solutions to the complete system of disk structure equations including advection and radial gradients have quite different properties at high $\dot{m}$ compared to the solutions of the standard disk model. For $\dot{m}<\dot{m}_{c r}=36$ (dashed lines), including the gradient terms gives rather small corrections to the standard

disk model. When $\dot{m}>36$ advection becomes essential and for $\dot{m}>\dot{m}_{c r}$ it changes the picture qualitatively. When $\dot{m}>\dot{m}_{c r}$ solutions do exist extending continuously from large radii to the innermost disk region 
(the solid lines) where the solution passes through a "sonic point". However for small values of the mass accretion rate $\dot{m}<0.1$ all types of solutions (solutions without advection, optically thick solutions with advection and solutions with advection and optical depth transition) are very similar and have the same structure.

Figure 3 shows the dependence of $\ell_{i n}$ on accretion rate $\dot{m}$ [left panel], and the locations of the inner singular points $\left(r_{s}\right)_{i n}$ as a function of $\dot{m}$ [right panel] for $\alpha=0.5$ and $M_{B H}=10 M_{\odot}$. Dushed line corresponds to the advective optically thick solutions, and solid line - to the advective solutions with optical depth transition.

Figure 4 shows the dependence of relation of gas pressure to the total pressure in accretion disk, $\beta_{s}$, on $\dot{m}$ at the inner singular points for the same models as in Figure 3. The line styles are the same as in Figure 3. The change of value of $\beta$ form 1 to 0 corresponds to the change of a state from the gas pressure to radiative pressure dominated one. The thin disks with $\beta \simeq 1$ are locally stable, whereas the parts of the disk in which $\beta \simeq 0$ could be thermally and viscously unstable at $\dot{m} \lesssim 100$ (Pringle, Rees, \& Pacholczyk 1973).

In Figures 5, 6, 7 we plot solutions with advection for $\dot{m}=48>\dot{m}_{c r}, \alpha=0.5$, and $M_{B H}=10 M_{\odot}$. We compare the solution obtained in optically thick approximation (dashed line), and the solution with optical depth transition (solid line).

In Figure 5 the the smooth transition between optically thick outer region of accretion disk and optically thin inner region when the accretion rate is higher than the critical one is demonstrated (the solid line).

Figure 6 shows the dependence of the temperature on the raduis for the same model as Figure 5 and the transition from the outer regions with relatively low temperature to the inner edge of disk with temperature close to the $10^{9} \mathrm{~K}$ is clearly seen (solid line).

The corresponding dependence of density on the raduis is shown on Figure 7 . When the optical depth transition formula is taken into account (solid line) the density of the disk decreases considerably in the inner regions of accretion disk, that corresponds to the optically thin high temperature state of the inner regions.

Using analysis discussed by Artemova et al. (2001) we have determined a type of singular points in our numerical solutions.

In the case of viscosity prescription (1) the solutions have two singular points. The inner singular points, $\left(r_{s}\right)_{i n}$, can be saddles or nodes depending on values of $\alpha$ and $\dot{m}$. But in the models discussed above, for $\alpha=0.5$ and $\dot{m}>1.0$, the inner singular point has always nodal-type. Note that the change of type from the saddle to the nodal one does not introduce any features in solutions. The outer singular points, $\left(r_{s}\right)_{o u t}$, are always of a saddle-type. In the case of viscosity prescription (2) the solutions have only inner singular points which are always of a saddle-type.

We have obtained unique solutions for structure of advective accretion disk with optical depth transition in a wide range of accretion rates and $\alpha$-parameters. Both viscosity prescriptions (1) and (2) have been investigated. The solutions corresponding to both prescriptions are very close for $\alpha \lesssim 0.1$, and begin to differ at larger $\alpha$. Due to the technical problems in calculation of the high viscosity models in the case of viscosity prescription (2) we consetrated our efforts on the study of high accretion rate models using prescription (1). 
The main difference of the present study from the previous ones is in using more sophisticated numerical technique which accurately treats the regularity conditions in the inner singular point of equations. We have performed an analytical expansion at the singular point to calculate the derivatives of physical quantities. These derivatives help us to find the proper integral curve passing through the singular point. Theis approach allow us to avoid numerical instabilities and inaccuracies, appearing when only variables at the singular point, but not its derivatives, are included into a numerical scheme.

\section{Acknowledgments.}

Yu.A. and G.B.- K. thank RFFI Grant 02-02-16900 for partial support of this work.

\section{References}

Abramowicz, M.A., Czerny, B., Lasota, J.P., \& Szuszkiewicz, E. 1988, ApJ, 332, 646

Abramowicz, M.A., Chen, X., Kato, S., Lasota, J.-P. \& Regev, O. 1995, ApJ, 438, L37

Artemova, I.V., Bisnovatyi-Kogan, G.S., Bjornsson, G.,\& Novikov, I.D. 1996, ApJ, 456, 119

Artemova, I.V., Bisnovatyi-Kogan, G.S., Igumenschev, I.V., \& Novikov, I.D. 2001, ApJ, 549, 1050

Chakrabarti, S.K. 1996, ApJ, 464, 664

Chen, X., \& Taam, R.E. 1993, ApJ, 412, 254

Chen, X. 1995, MNRAS, 275, 641

Chen, X., Abramowicz, M.A., Lasota, J.-P., Narayan, R. \& Yi, I. 1995, ApJ, 443, L61

Liang, E.P., \& Thompson, K.A. 1980, ApJ, 240, 271

Liang, E.P. \& Wandel, A. 1991, ApJ, 376, 746

Luo, C. \& Liang, E.P. 1994, MNRAS, 266, 386

Matsumoto, R., Kato, S., Fukue, J., \& Okazaki, A.T. 1984, PASJ, 36, 71

Misra, R. \& Melia, F. 1996, ApJ, 465, 869

Muchotrzeb, B., \& Paczyński, B. 1982, Acta Astr., 32, 1

Muchotrzeb, B. 1983, Acta Astr., 33, 79

Muchotrzeb-Czerny, B. 1986, Acta Astr., 36, 1

Narayan, R. \& Yi, I. 1994, ApJ, 428, L13

Narayan, R. 1996, in "Basic Physics of Accretion Discs", ed. S. Kato et al., (New York : Gordon and Breach)

Paczyński, B., \& Wiita, P.J. 1980, A\&A, 88, 23

Paczyński, B., \& Bisnovatyi-Kogan, G.S. 1981, Acta Astr., 31, 283

Shakura, N.I. 1972, Astron. Zh., 49, 921

Shakura, N.I., \& Sunyaev, R.A. 1973, A\&A, 24, 337

Wandel, A. \& Liang, E.P. 1991, ApJ, 380, 84 


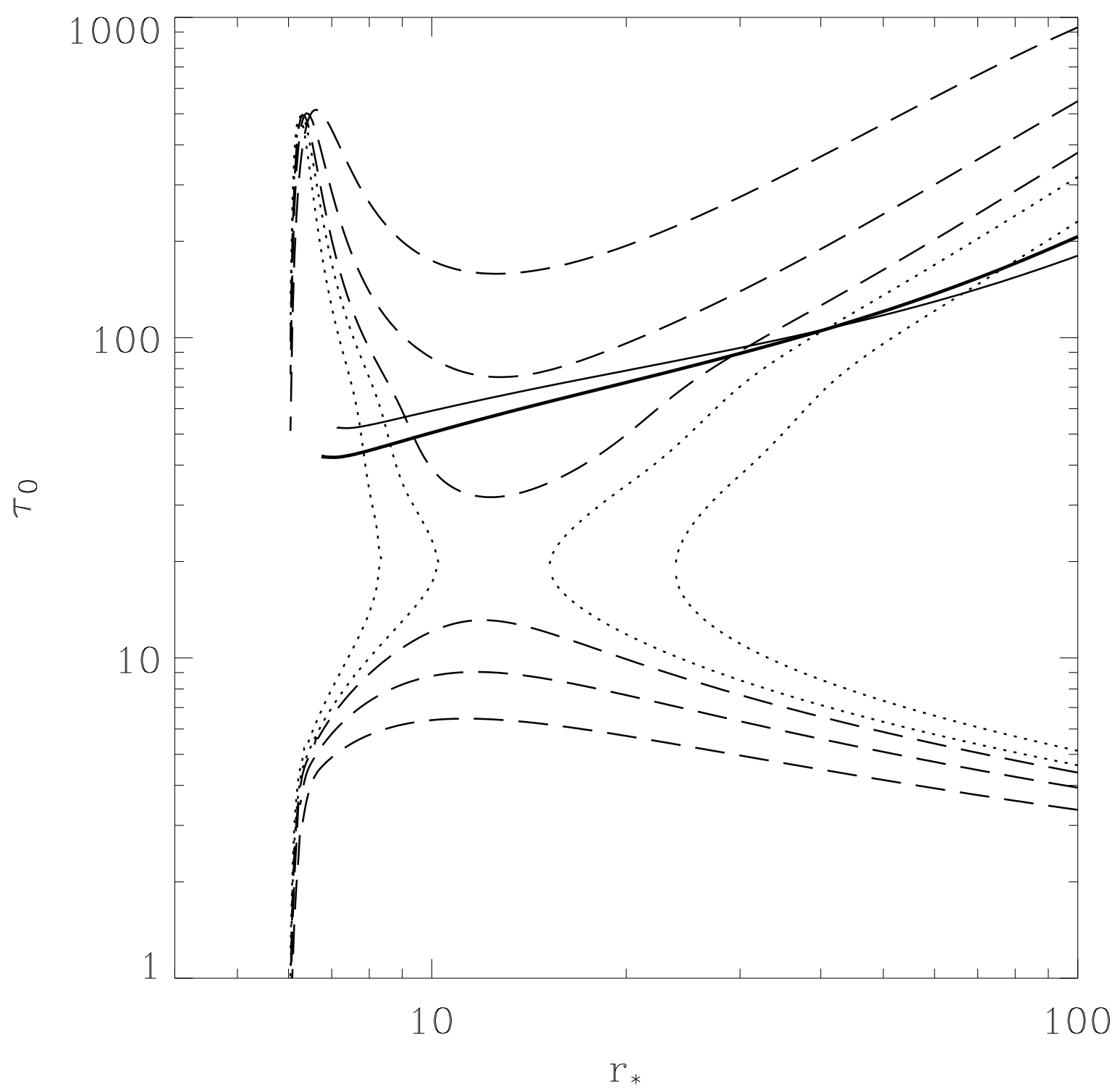

Fig. 1. - The dependence of the Thomson scattering depth on the radius for the models with $\alpha=0.5$ and $M_{B H}=10 M_{\odot}$. Dashed lines correspond to the solutions without advection and $\dot{m}<\dot{m}_{c r}=36$. Dotted lines correspond to the non-physical solutions without advection for $\dot{m}=\dot{m}_{c r}=36$ and $\dot{m}=50$ (from the center to the edge of the picture respectively). Solid lines correspond to the solutions with advection and the mass accretion rate higher than the critical one. Thick solid line corresponds to $\dot{m}=36.0$, and the thin solid line to $\dot{m}=50.0$. 


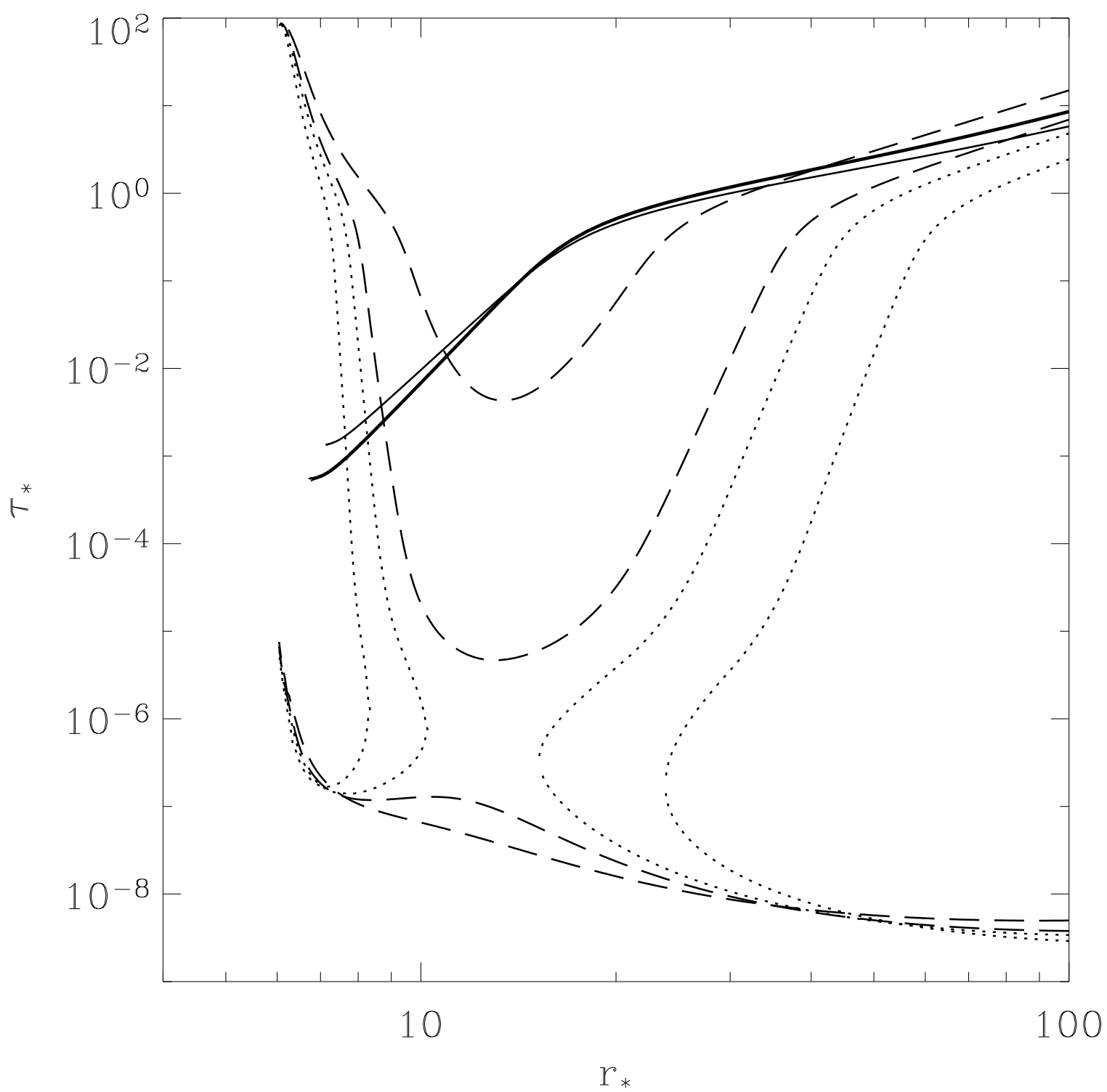

Fig. 2.- The dependence of the effective optical depth on the radius for the same models as in Fig.1. The styles of the curves are the same as in Fig.1. 

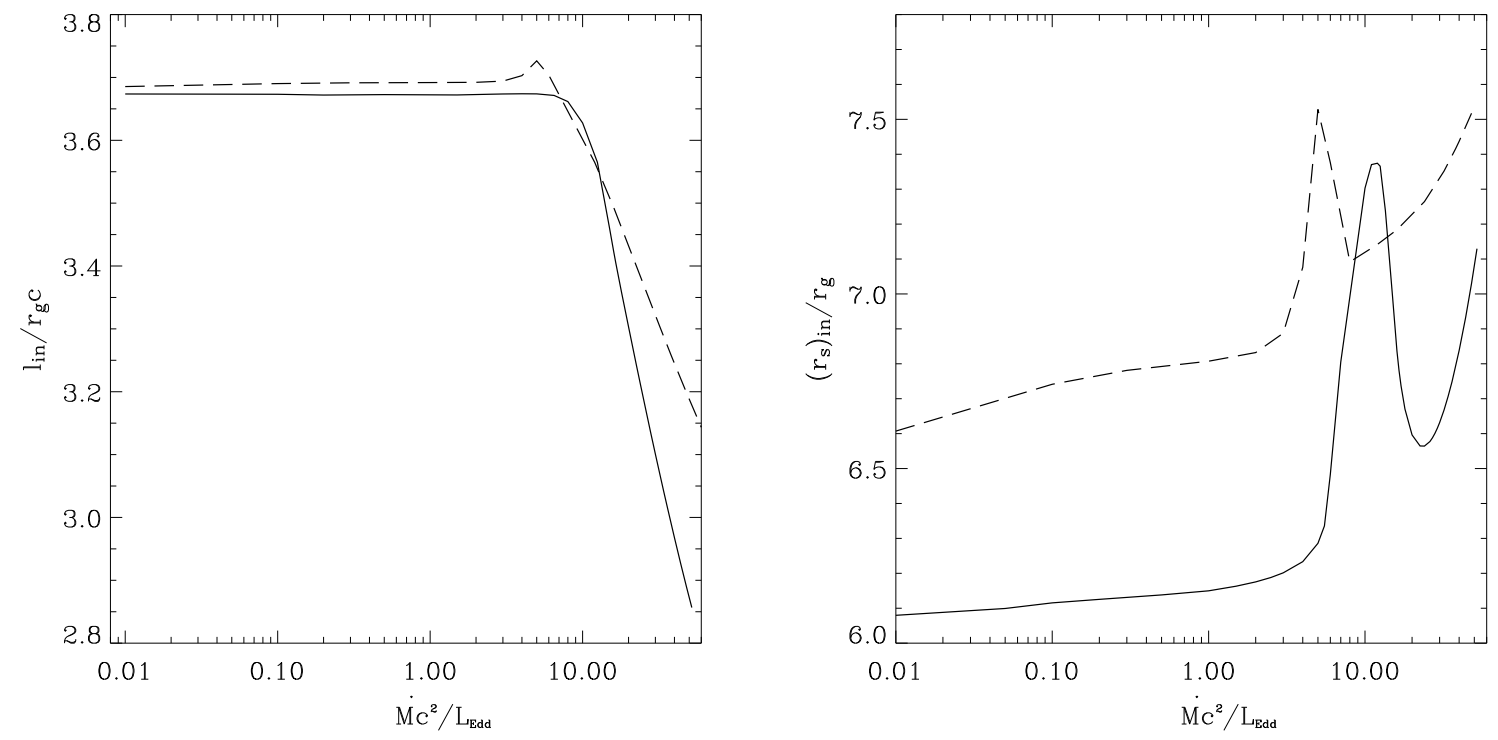

Fig. 3.- The specific angular momentum $\ell_{i n}$ (left panel) and the position of the inner singular points (right panel) as a function of the mass accretion rate $\dot{M}$ for viscosity parameters $\alpha=0.5$ and $M_{B H}=10 M_{\odot}$. The solid lines represent models with the optical depth transition and advection, whereas the dashed lines correspond to advective optically thick ones. 


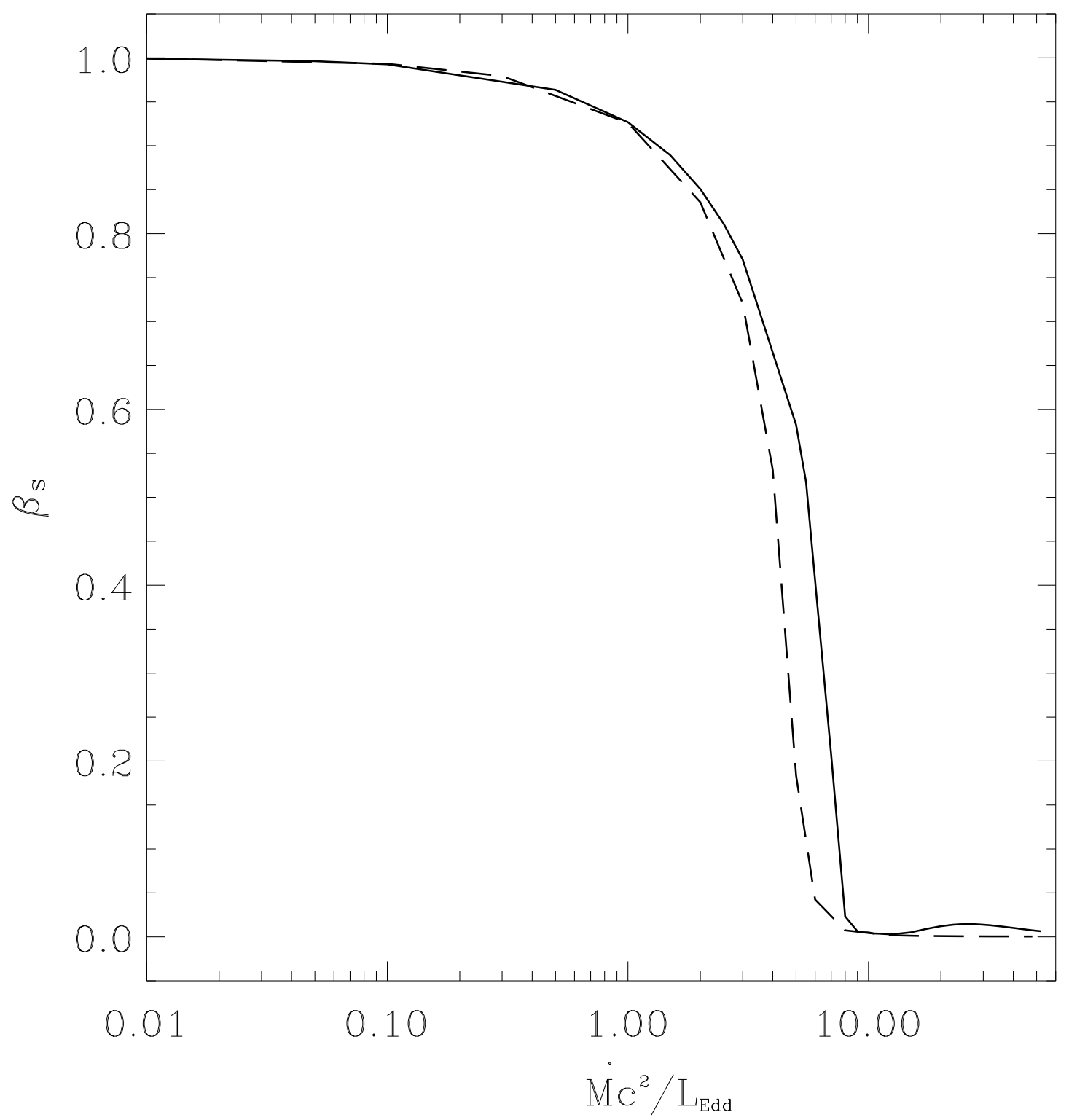

Fig. 4.- Ratio of the gas pressure to the total pressure at the inner singular point, $\beta_{s}$, as a function of the mass accretion rate $\dot{M}$. Models with $\alpha=0.5$ and $M_{B H}=10 M_{\odot}$ are shown. The styles of the curves are the same as in Fig.3. 


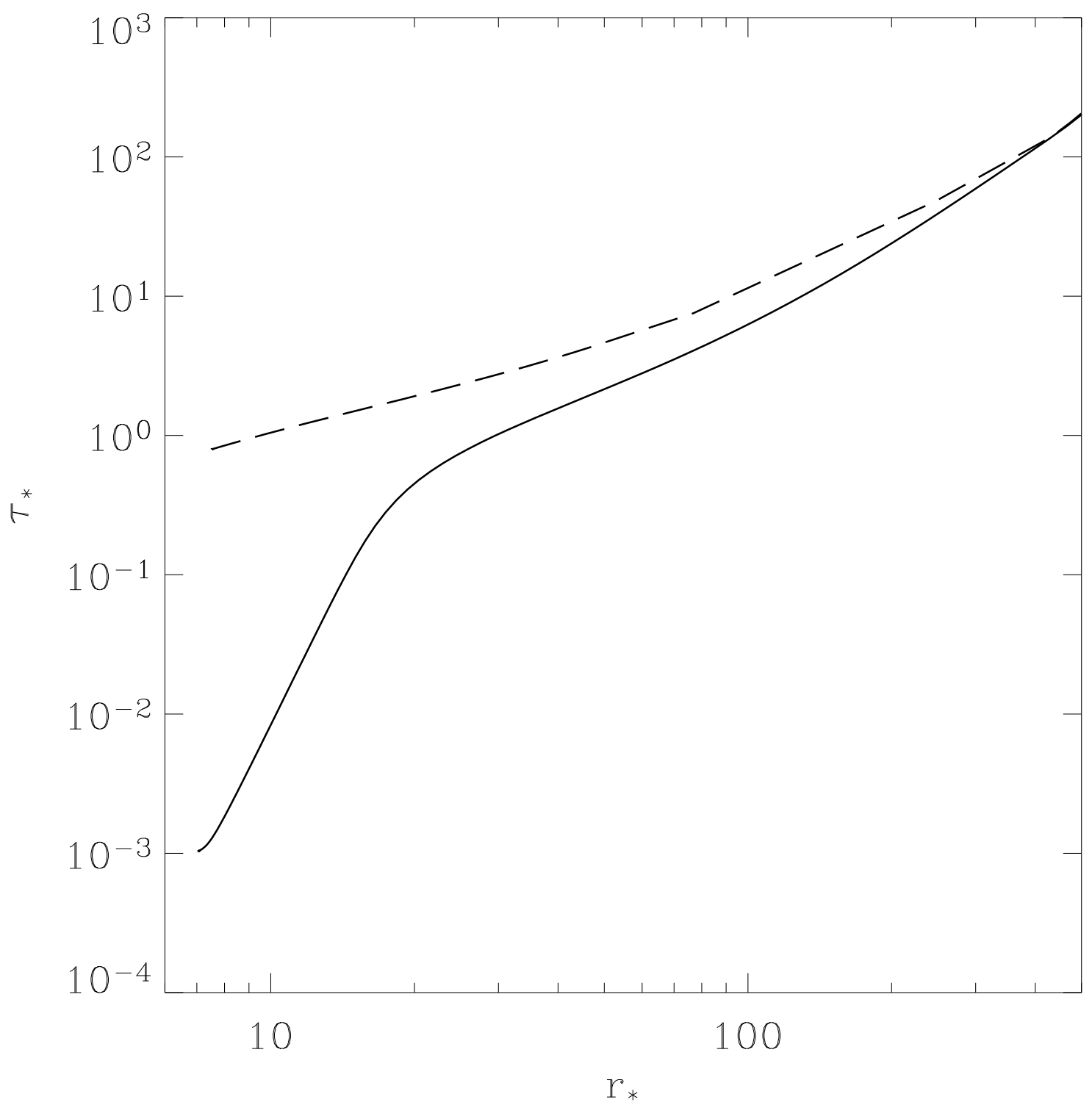

Fig. 5. - The dependence of the effective optical depth on the radius for $M_{B H}=10 M_{\odot}, \dot{m}=48, \alpha=0.5$. Dashed line corresponds to the solution with advection and without optical depth transition, solid line corresponds to the solution with advection and optical depth transition. 


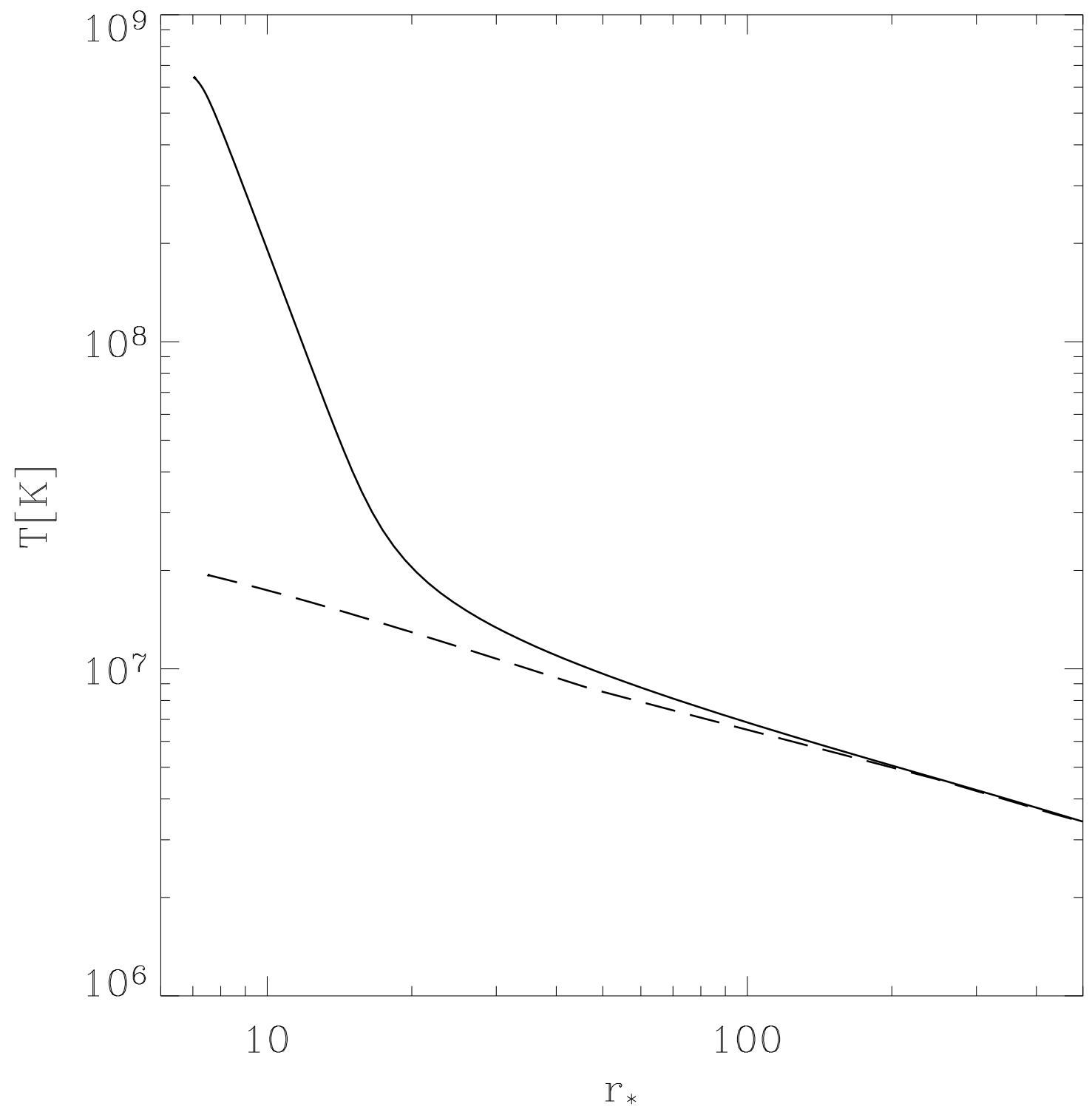

Fig. 6. - The dependence of the temperature on the radius for the same models as in Fig.5. The styles of the curves are the same as in Fig.5. 


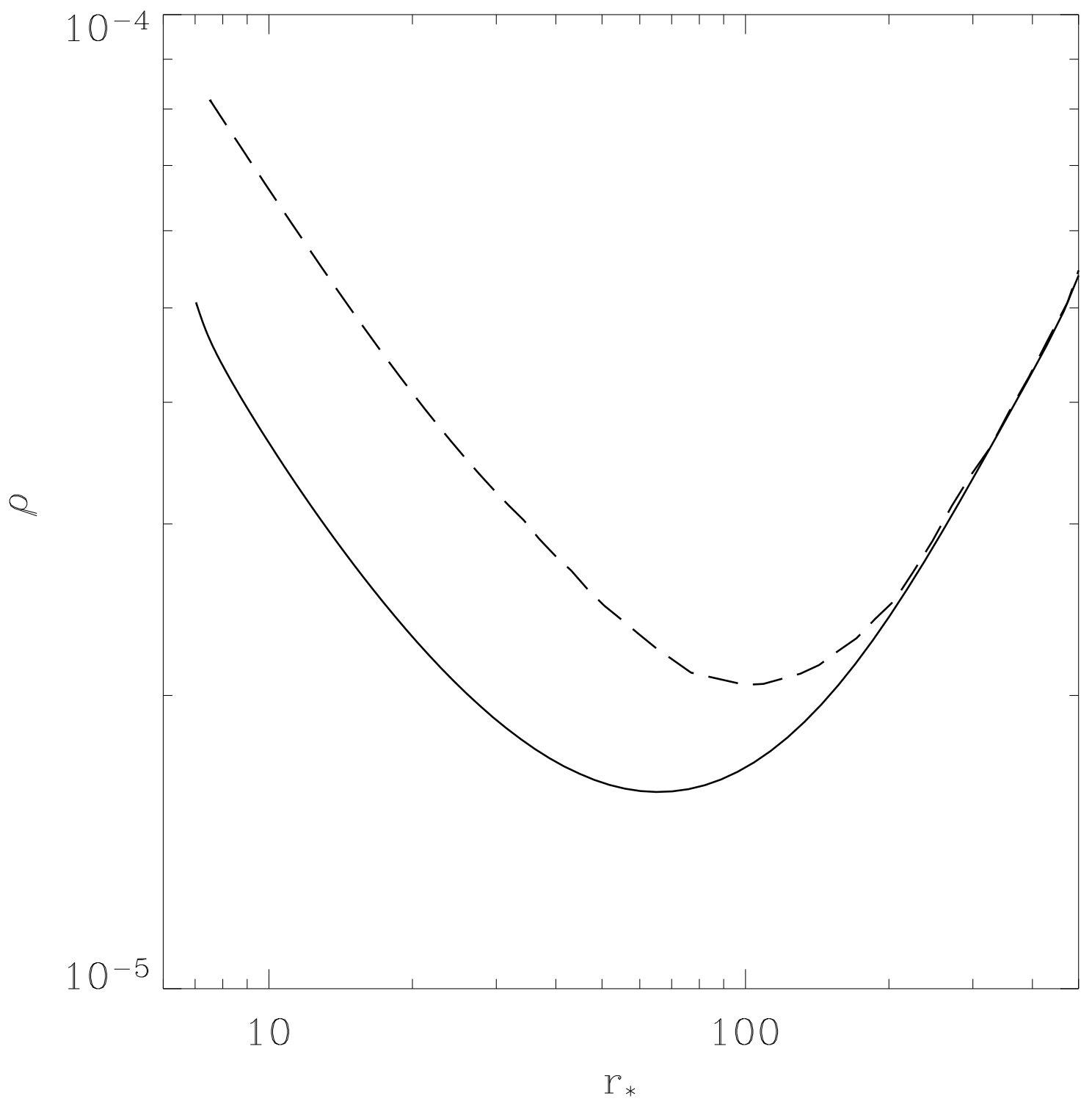

Fig. 7.- The dependence of the density on the radius for the same models as in Fig.5. The styles of the curves are the same as in Fig.5. 